\title{
RESIDUAL CAPACITY PREDICTION OF BLAST-LOADED STEEL COLUMNS USING PHYSICS-BASED FAST RUNNING MODELS
}

\author{
L.K. STEWART ${ }^{1} \&$ K.B. MORRILL ${ }^{2}$ \\ ${ }^{1}$ School of Civil and Environmental Engineering, Georgia Institute of Technology, USA. \\ ${ }^{2}$ Karagozian and Case Structural Engineers, USA.
}

\begin{abstract}
The implementation of models for the prediction of structural behavior due to blast loads has become vital in the evaluation of threats. Most often, these models require complex finite element analysis and a background in structural and blast engineering to use effectively. Although the finite element models (FEMs) are robust and accurate, it is often necessary to evaluate structures and their potential risks in a relatively short time frame, much quicker than the time a complex, non-linear analysis would necessitate. Typically, simplified engineering tools, such as single degree of freedom models, are utilized in these scenarios but, while effective for some blast applications, often lack the fidelity necessary to sufficiently represent the variable (spatial and temporal) loading and non-linear response of the structure from a range of explosive events.

This paper presents a methodology for a blast analysis tool whereby a FEM is used in conjunction with an artificial neural network to develop a physics-based fast running model. The methodology, which can be applied to a variety of engineering problems, is applied to residual capacity predictions for steel columns subjected to a range of vehicle-borne explosive threat scenarios. Through a set of validation scenarios, the model is shown to be an effective blast analysis tool capable of predictions of structural response within seconds and accessible for security and engineering professionals with varying technical backgrounds.

Keywords: artificial neural network, blast, steel columns, structural models.
\end{abstract}

\section{INTRODUCTION}

The majority of fast running models (FRMs) for blast analysis heavily rely on single degree of freedom (SDOF) systems [1]. In an SDOF blast analysis, a column with length, $L$, displacement, $x$, and applied blast load, $w(t)$, is considered and is shown in Fig. 1. For simplicity, the blast load is typically chosen to be uniform and the boundary conditions are modified to represent the configurations of the structure. The column, which has a continuous displaced shape along the length, is simplified into a spring-mass system, in which the equivalent mass of the structure, $M$, is lumped into a single element with an applied time-varying load, $F(t)$. The resistance of the column, $R$, which is a function of the displacement of the mass, $x$, is also incorporated and found using the basic stiffness and boundary conditions of the structure. The displacement is computed by numerically solving the equation of motion.

The displacement computed from the SDOF is typically defined as the displacement at the mid-span of the column. The predictions using SDOF methods are satisfactory for a wide range of blast problems; however, the methodology does directly yield information as to the response of the column such as capacity [2,3] or type of damage. Additionally, because it assumes a uniform blast loading along the height and the width, it is not able to account for the sometimes extreme variability in pressure and impulse along the height of the column for near-field charges. Such explosive scenarios are more likely to cause localized behaviors and failures such as web buckling, flange deformations, and rupture of the web [4], none of which can be described or predicted using current SDOF model methodology. 

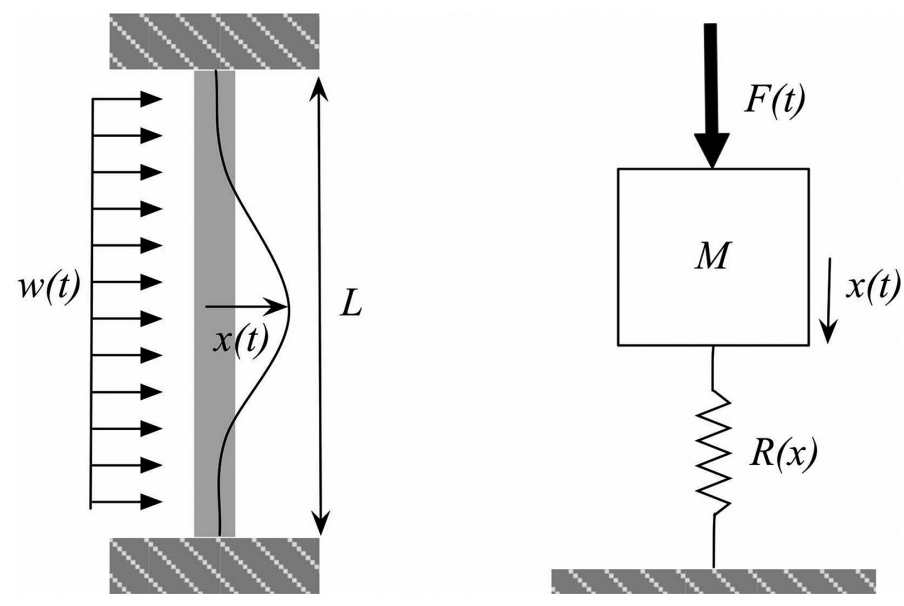

Figure 1: SDOF model for blast loading.

To accurately predict the column response of this nature, it is often necessary to employ a validated finite element model (FEM) that can sufficiently capture behaviors seen in blast-damaged columns. Such models, although accurate, can involve millions of elements and can be extremely time consuming to compute, which is undesirable in many cases. To be effective, the tool must be able to accurately predict the column response for any subset of given conditions and parameters in a relatively short time frame. For this reason, an artificial neural network (ANN), discussed in the following sections, was used to process the parameters and predict the capacity response using data generated from the high-fidelity FEMs.

The model developed in this paper is presented for the prediction of residual column capacity subjected to vehicle-borne threats. The columns are loaded in both the strong and weak directions and included freestanding columns with no beam connections or base plates. Column size considered ranged from W8 to W14 steel wide flanges. It is meant to be the starting point for multiple variations and additions as the methodology described is pertinent to applications of other column configurations and other types of structures.

\section{ARTIFICIAL NEURAL NETWORKS}

An ANN is a mathematical or computational model that tries to simulate how biological nervous systems, such as the brain, process information. In the model, each single artificial neuron, shown in Fig. 2a, accepts information from a number of given inputs. Each of those inputs comes with a strength (or weight) that corresponds to the synaptic efficiency in a biological neuron. Each neuron has a corresponding threshold or bias value, $b$. The weighted sum of the inputs is computed and subtracted from the threshold value to compute the activation value, $a$. This activation value is passed through an activation function (transfer function), $f$, to produce the output of the neuron.

A network of neurons is composed of many single element neurons operating in parallel. The connections between the elements are often interconnected by multiple inputs and multiple layers of hidden neurons, which can produce multiple outputs. One example of a network of interconnected neurons is given in Fig. 2b. For the purpose of this paper, a feed-forward network, which passes information in one direction, with one layer of hidden neurons and one output parameter will be used and discussed further. Many references discuss ANNs in more detail and provide information on additional types of neural network structures $[5,6]$. 
(a)
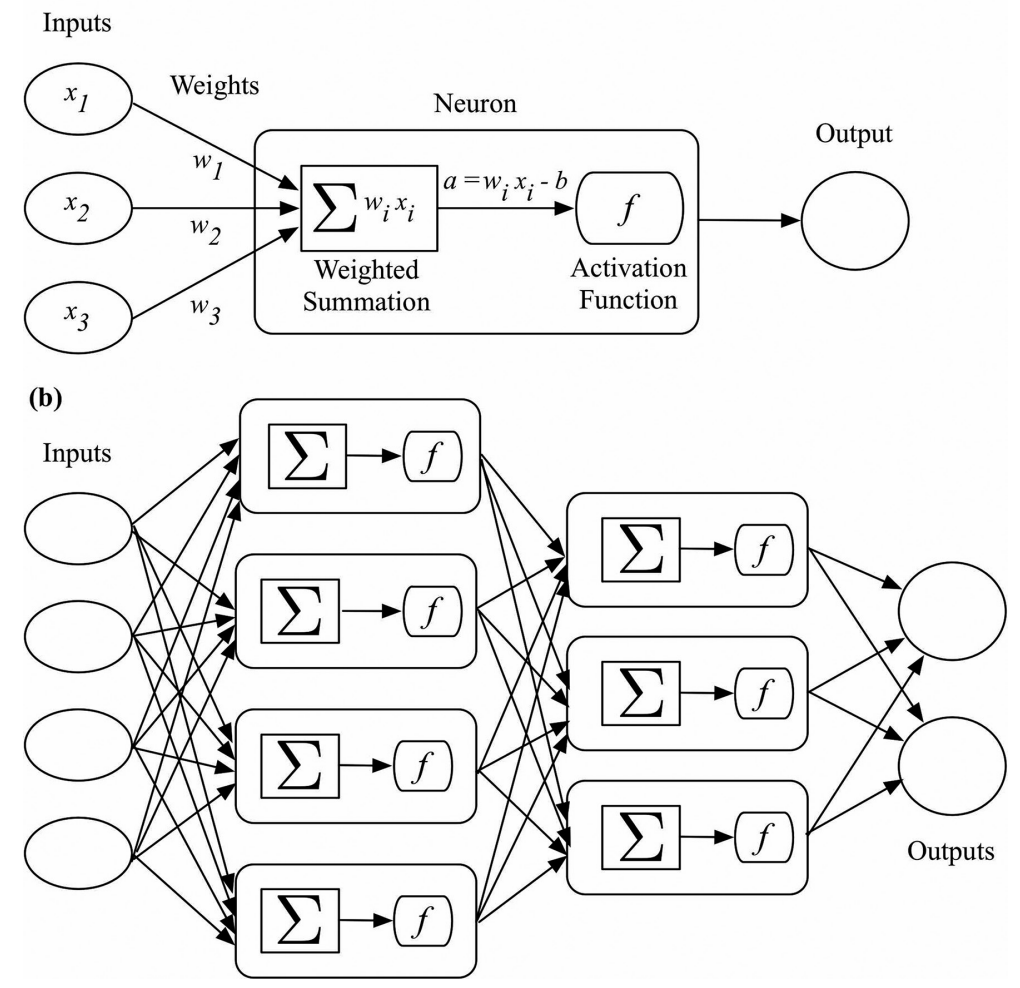

Figure 2: Single artificial neuron model (a) and artificial neural network (b).

The procedure of modifying the weights and biases of the network to perform some particular task, such as predicting column capacity, is done with a training algorithm. The training algorithm can be supervised or unsupervised. This methodology will use a supervised algorithm whereby the inputs and outputs of the training sample set are known and applied to the model. In this case, the inputs are applied to the network and outputs are computed. These calculated outputs are compared with the actual target values, and the weight and bias values are updated.

There are many training algorithms that can be used to help the model 'learn'. The majority of the algorithms are methods that seek to provide a numerical/iterative method for the minimization of the error function created over the space of parameters. Some examples of these types of algorithms are the back propagation method, the conjugate gradient descent method, the quasi-Newton method, and the Levenberg-Marquardt method. Once acceptable values from the weights and biases have been determined, the model can then be used to predict outputs from another set of desired inputs.

\section{SELECTION OF PARAMETERS}

The input parameters for the ANN were divided into two categories: loading parameters and structural parameters. The loading parameters were needed to adequately describe the loads imparted on the column by a typical vehicle bomb. The loading environment can be described by a charge of a given weight, $W$, at a given standoff, $R$. Typical vehicle bombs considered are 


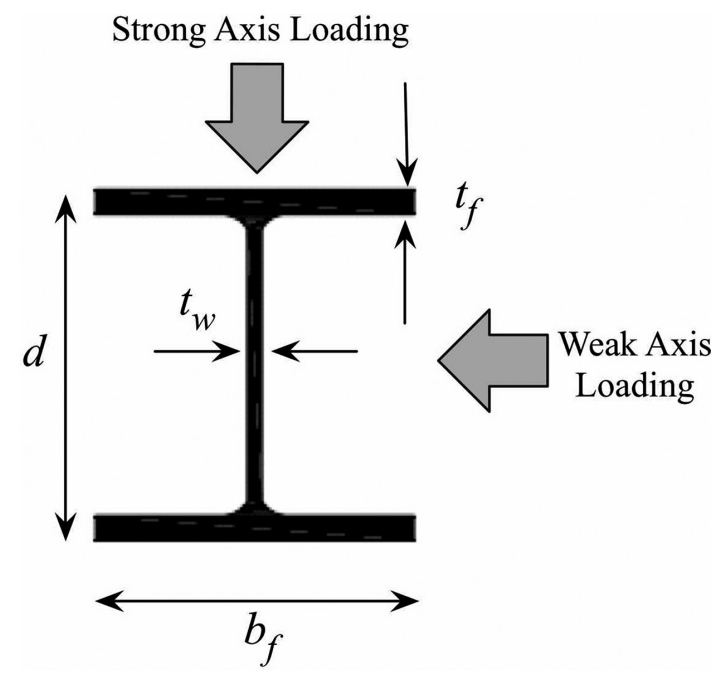

Figure 3: Typical wide flange dimensions.

within the range of 500-1500 lbs (226-680 kg) [7] and are normally located a few feet off the ground. For this study, a constant height of $3 \mathrm{ft}(0.9 \mathrm{~m})$ from the ground was selected. The standoffs of interest include vehicles at the curb and are within the range of 3-25 ft (0.9-7.6 $\mathrm{m})$ from the column. Any standoff closer than $3 \mathrm{ft}(0.9 \mathrm{~m})$ is considered a near-contact charge and is outside the scope of this paper. Standoffs outside of $25 \mathrm{ft}(7.6 \mathrm{~m})$ are not included because the damage to the column would be minimal and SDOF methods are likely to be sufficient. For security purposes, charge weight and standoff are normalized in this paper through the scaled distance, $Z$, as given in the following equation:

$$
\mathrm{Z}=\mathrm{R} / \mathrm{W}^{1 / 3}
$$

The structural parameters were chosen to sufficiently describe the geometry of a steel wide flange section [8]. The geometry of a generic section is shown in Fig. 3. Parameters and the range of values were chosen based on applicability to blast design and on the availability of data to validate the FEM. The first parameter considered is the column depth, $d$, which is an important parameter because as $d$ increases, the tributary area for weak axis loading increases which, in turn, increases the load on the column. In the strong axis direction, it also contributes to the stiffness in the loaded direction. Column depths of interest are those associated with W8-W14 sections and range from 7.93 to 22.84 inch $(20.1-58.0 \mathrm{~cm})$.

The second structural parameter of interest is the width-thickness ratio of the depth, $d$, to the web thickness, $t_{\mathrm{w} .}$ The parameter will, therefore, be defined as $d / t_{\mathrm{w}}$. The ranges for this parameter for columns of W8-W14 are 6.11-37.04. Data used for model calibration solely involved relatively square columns; therefore, it will be assumed that the columns used in this analysis will also be relatively square with $\mathrm{b} / \mathrm{d}_{f}>0.75$. The length of the flange, $\mathrm{b}_{\mathrm{f}}$, will therefore not be used as a parameter and can be considered to be equal to $d$ in any subsequent calculations used for model training.

The next structural parameter involves the flange thickness, $t_{\mathrm{f}}$. The thickness of the web, $t_{\mathrm{w}}$, will be included in a ratio with the thickness of the flange. The thickness ratio, $t_{\mathrm{f}} / t_{\mathrm{w}}$, is appropriate because, among other things, it specifically defines the amount of local and global damage of the column depending on its orientation. For example, in a strong axis configuration, if the flange thickness is small compared to its web, more local flange deformation 
Table 1: Model parameters and ranges.

\begin{tabular}{lll}
\hline Type & Parameter & Range \\
Loading & Scaled Distance, $\mathrm{Z}$ & 0 to 10 \\
Structural & Depth, $\mathrm{d}$ & 7 in to $25 \mathrm{in}(20$ to $58 \mathrm{~cm})$ \\
Structural & Width-Thickness Ratio, $d / t_{\mathrm{w}}$ & 6.1 to 37.0 \\
Structural & Thickness Ratio, $t_{\mathrm{f}} t_{\mathrm{w}}$ & 1.3 to 1.8 \\
Structural & Length, $\mathrm{L}$ & 8 to $25 \mathrm{ft}(2.4$ to $6.1 \mathrm{~m})$ \\
Output & Column Capacity Ratio, CCR & 0 to 1 \\
\hline
\end{tabular}

would occur over the global deformation of the column that is more governed by the web. Typical thickness ratios range from 1.62 to 1.77 .

The last structural parameter is the column length, $L$. The column length is significant because as the column height increases, the amount of deformation increases due to an increase of tributary area, as well as the buckling length. Column lengths appropriate for this model range from 8 to $20 \mathrm{ft}(2.4-6.1 \mathrm{~m})$ as found in a typical building. A summary of the input parameters and their ranges are included in Table 1.

The residual column capacity was selected to be the output parameter of interest in this example model. Other output parameters can be selected and considered simultaneously using the ANN if desired. The residual capacity is quantified by the column capacity ratio (CCR). The CCR is defined as the ratio of the residual column capacity after blast damage over the virgin capacity of the column as described in eqn (2). For example, consider the case of a column with load-carrying capacity of $800,000 \mathrm{lbs}(363,000 \mathrm{~kg})$. The structural and loading parameters of that column and charge weight would be entered into the model and, for example, a CCR of 0.75 would be output. The CCR of 0.75 would indicate that the column could carry $75 \%$ of the initial load-carrying capacity of $800,000 \mathrm{lbs}(363,000 \mathrm{~kg})$, which would mean that the damaged column could carry up to $600,000 \mathrm{lbs}(272,000 \mathrm{~kg})$. If the column were designed to hold 700,000 $(317,000 \mathrm{~kg})$, the column would likely fail after the blast, since it could only carry $600,000 \mathrm{lbs}(272,000 \mathrm{~kg})$

$$
C C R=\frac{\text { Blast damaged column capacity }}{\text { Virgin column capacity }}
$$

\section{SELECTION OF TRAINING POINTS}

The selection of individual training points was performed in an iterative process. An initial set of training points was selected and is described in this section. The initial set consisted of 51 samples in both the strong and the weak axis model. These samples each included a different combination of the five parameters described in the previous section. For each parameter, a nominal, minimum and maximum value was assigned based on the distribution and range of values for that parameter. The nominal value is selected as the most representative value of the sample. This can be based on mean, median, distribution, etc. and often times experience aids in the selection.

The nominal charge weight, $W$, was chosen to be $1,000 \mathrm{lbs}(454 \mathrm{~kg})$. The minimum was $500 \mathrm{lbs}(226 \mathrm{~kg})$ and the maximum was $1,500 \mathrm{lbs}(680 \mathrm{~kg})$. The minimum standoff, $R$, was set at $5 \mathrm{ft}(1.5 \mathrm{~m})$, the nominal was defined as $10 \mathrm{ft}(3 \mathrm{~m})$ and the maximum was set as $20 \mathrm{ft}$ 


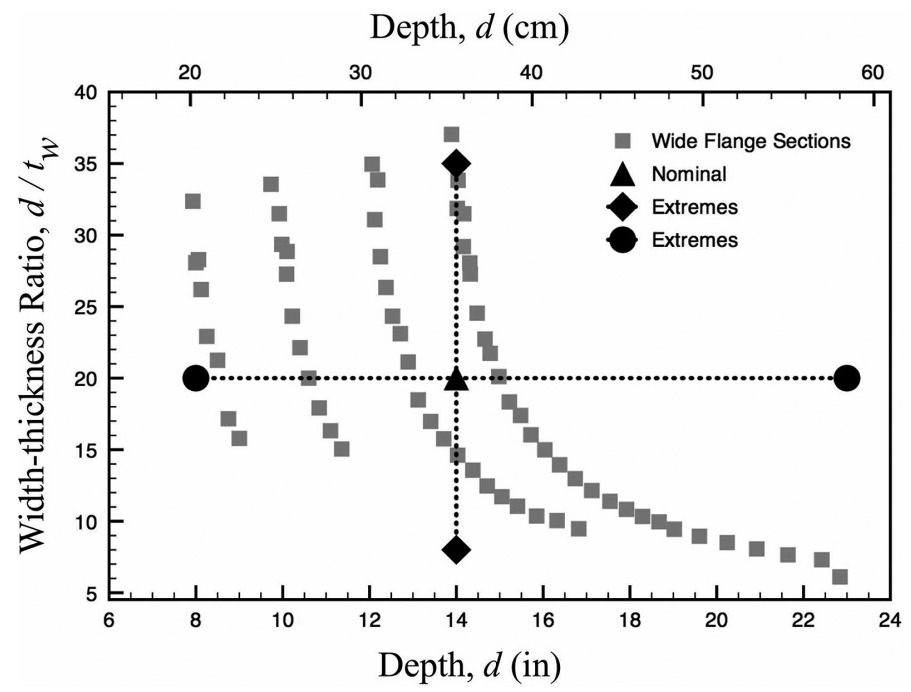

Figure 4: Depth versus width-thickness ratios.

$(6.1 \mathrm{~m})$. Since scaled distance $Z$ is used as the parameter in this paper, these values correspond to a nominal $Z$ of 1 with minimum and maximum 0.62 and 1.75 values, respectively. It should be noted that these are not the full minimum and maximum ranges because the model is able to extrapolate, to some extent, outside the minimum and maximum ranges defined.

This model considers W8-W14 sized columns with $b / d_{f}>0.75$. The corresponding depths, $d$, and width-thickness ratio, $d / t_{\mathrm{w}}$, associated with these sections are plotted in Fig. 4 . From the distribution of this plot, it was decided that a nominal depth of 14 inch $(35.6 \mathrm{~cm})$ would be used with a minimum and maximum of 8 inch $(20.3 \mathrm{~cm})$ and $22 \mathrm{inch}(55.9 \mathrm{~cm})$, respectively. Eight inches $(20.3 \mathrm{~cm})$ were chosen as the minimum for the width-thickness ratio, 20 inch $(50.8 \mathrm{~cm})$ for the nominal and $35 \mathrm{inch}(88.9 \mathrm{~cm})$ for the maximum.

Figure 5 gives a plot of the depth, $d$, versus the thickness ratio, $t_{f} / t_{\mathrm{w}}$. In this case, the nominal for the thickness ratio parameter was chosen as 1.60 because that value seemed to best represent the distribution of points. The minimum and maximum were selected as 1.45 and 1.75 , respectively.

The last parameter is the column length, $L$. A minimum height was chosen as $8 \mathrm{ft}(2.4 \mathrm{~m})$ and a maximum was chosen as $20 \mathrm{ft}(6.1 \mathrm{~m})$. The nominal was decided to be $12 \mathrm{ft}(3.7 \mathrm{~m})$ because that could be considered an average height for typical structures. A summary of all the parameters and their values is included in Table 2.

The parameters were combined in a systematic manner for the initial data set. The five parameter combinations span a five-dimensional space that is difficult to visualize. For this reason, a three-dimensional data set will be used for explanation purposes. If a three-parameter space, $x_{1}, x_{2}, x_{3}$, is considered and plotted as a cube with the nominal of each value at the center of the axis and the minimum and maximum as the two extremes, as shown in Fig. $6 \mathrm{a}$, then the center black dot would correspond to the sample with $x_{1}=$ nominal, $x_{2}=$ nominal, and $x_{3}=$ nominal. The next set of points is found by varying one parameter to an extreme and keeping the remaining parameters fixed and nominal. For example, $x_{1}=$ minimum, $x_{2}=$ nominal, and $x_{3}=$ nominal. These points are shown in the cube in Fig. $6 \mathrm{~b}$ as the grey squares. For a given number of parameters, $n$, the number of samples corresponding 


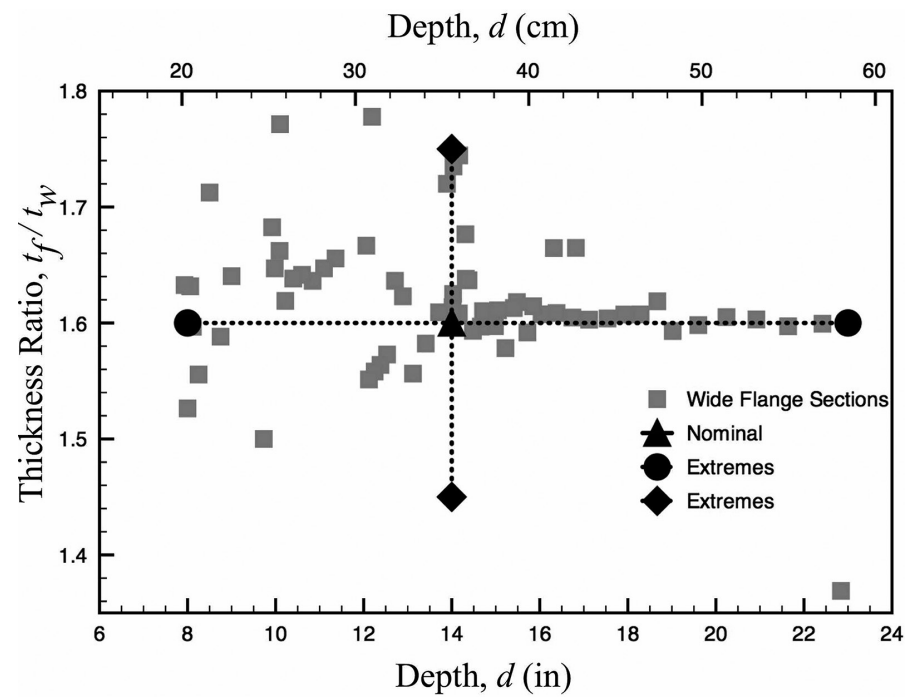

Figure 5: Depths versus thickness ratios.

Table 2: Summary of nominal and extreme parameter values.

\begin{tabular}{llll}
\hline Parameter & Minimum & Nominal & Maximum \\
\hline Scaled Distance, $Z$ & 0.62 & 1 & 1.75 \\
Depth, $d$ & 8 in $(20.3 \mathrm{~cm})$ & 14 in $(35.6 \mathrm{~cm})$ & 22 in $(50.8 \mathrm{~cm})$ \\
Width-Thickness Ratio, $d / t_{\mathrm{w}}$ & 8 & 20 & 35 \\
Thickness Ratio, $t_{f} / t_{w}$ & 1.45 & 1.60 & 1.75 \\
Length, $L$ & $8 \mathrm{ft}(2.4 \mathrm{~m})$ & $12 \mathrm{ft}(3.7 \mathrm{~m})$ & $20 \mathrm{ft}(6.1 \mathrm{~m})$ \\
\hline
\end{tabular}

(a)

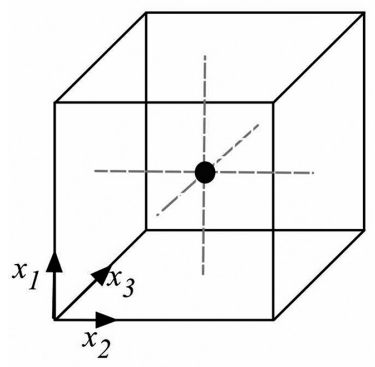

Nominal Case (b)

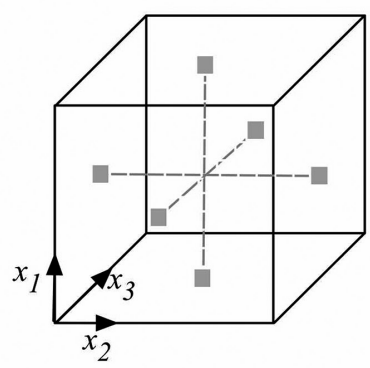

One Extreme Parameter (c)

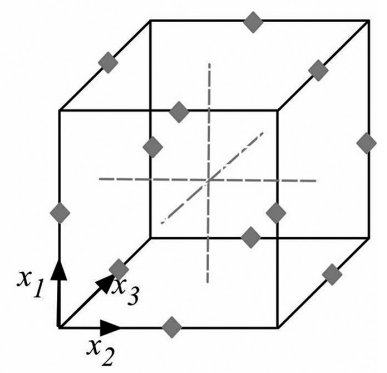

Two Extreme Parameters

Figure 6: Three-dimensional sample space: (a) nominal case, (b) one extreme parameter, and (c) two extreme parameters. 
to varying one parameter is $2 n$. The last set of points used in this initial data set is found by varying two parameters in all combinations of extremes and keeping the third parameter fixed as nominal. For example, $X_{1}=$ minimum, $X_{2}=$ maximum, and $X_{3}=$ nominal. These points are shown in the cube in Fig. $6 \mathrm{c}$ as the dark grey diamonds. For a given number of parameters, $n$, the number of samples corresponding to varying two parameters is $2 n(n 1)$. If all the above-mentioned samples are considered, then the total number of initial samples, $S$, for $n$ parameters is given by eqn (3). For the five input parameters in this model, the number of initial samples is 51 .

$$
S=1+2 n^{2}
$$

\section{GENERATION OF DATA SET}

The data used in this model were generated using an FEM similar to that which was validated for strong and weak axis steel columns tested experimentally [9]. The model was simplified specifically in the boundary conditions to make for a more realistic scenario and also to allow for a faster run time of the model. Details regarding the two models and the loading conditions are described in the next two sections.

\subsection{Strong axis model}

The FEM model developed for training calculations is shown in Fig. 7 using LS-DYNA, a non-linear, explicit finite element software [10]. The model is capable of capturing a variety of loading conditions and configurations within one generalized model. The model is also adaptable to the creation of additional parameters such as beam/column connections, boundary condition variations and preload, allowing the data generated in this research to help train any future models.

The column mesh dimensions and geometry are generalized according to the FRM input parameters. The model is made with shell elements consistent in size and material with those used in the validated model [9]. The column length, $L$, is defined as the clear length between the supports, which are modeled by solid element support pads that have dimensions which are functions of the column geometry. The column was left continuous through the supports both top and bottom to allow the column web and flange to deform near the supports. This was also done to represent the case of a typical multistory construction, where the columns run continuous between floors and often go below ground level to basement floors.

The column boundary conditions were chosen primarily to avoid an over-constraining of the column, which would be dominated by flexural response. They were chosen to allow the local shear and buckling deformation to occur that were observed in various field tests. Roller boundary conditions were applied to the solid element pads to prevent horizontal and vertical translations during loading and during rebound. A roller support was also placed at the bottom of the column to prevent vertical uplift. Node sets were placed at all boundary pads and supports to allow for the future restraint of the model if a fixed condition or spring addition is later desired.

For the strong axis runs, the load was applied to the flange opposite the support boundary as shown in Fig. 7. The loads were determined using BlastX [11] and were distributed over the height and width of the column allowing for a non-uniform load using a software developed during this research to apply a varying time-dependent load onto each element of the FEM. The loads did not take into account any clearing effects $[12,13]$ and therefore are considered to be conservative if clearing effects are appropriate. 

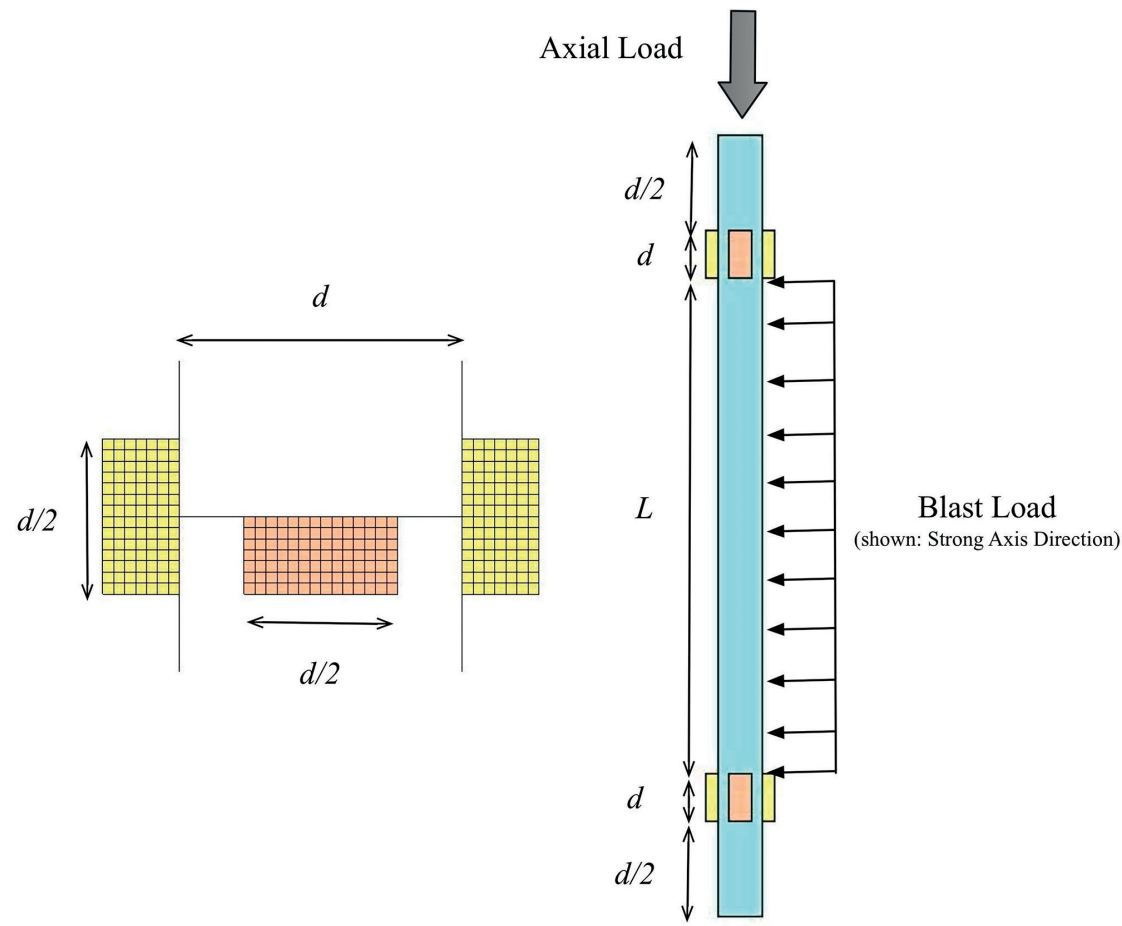

Figure 7: LS-DYNA finite element model.

The blast load was applied as a pressure load along the height of the column at time, $t=0$. The column was allowed to respond to the blast load for $300 \mathrm{~ms}$. Additional cases were run with a longer time frame, but it was found that it did not have a dramatic effect on the results. After $300 \mathrm{~ms}$, an axial load was applied in the form of a displacement-controlled boundary condition at a rate of $12 \mathrm{inch} / \mathrm{s}(30.5 \mathrm{~cm} / \mathrm{s})$. For the virgin capacity, the blast load was omitted and the axial load was applied at the same loading rate, with the hope that any rate effects from the high loading rate would nearly cancel out.

The 51 FEM cases were run according to the loading described. Results from a representative case are given in Fig. 8. The first set of results is from the case where the standoff was $10 \mathrm{ft}$, the column had a depth of 8 inch $(20.3 \mathrm{~cm})$, a width-thickness ratio of 20, a thickness ratio of 1.368 and a $12 \mathrm{ft}(3.7 \mathrm{~m})$ length. A summary of the behavior through loading is given in Fig. 8,left. The first two frames show the column being loaded by the blast load. The third frame shows the column being initially loaded axially and the last frame gives the column buckling due to the damage at the base (from an alternate view). The corresponding force diagram from this sample is given in Fig. 8(right). From this plot, the capacity of the column at failure was $297,000 \mathrm{lbs}(1,321 \mathrm{kN})$. This is compared to a virgin capacity test of 829,000 lbs $(3,687 \mathrm{kN})$. The associated CCR is 0.358 .

\subsection{Weak axis model}

The FEM used for the weak axis direction FRM is identical to that of the strong axis model described in above except for the weak axis runs, another support pad is used. For the weak 

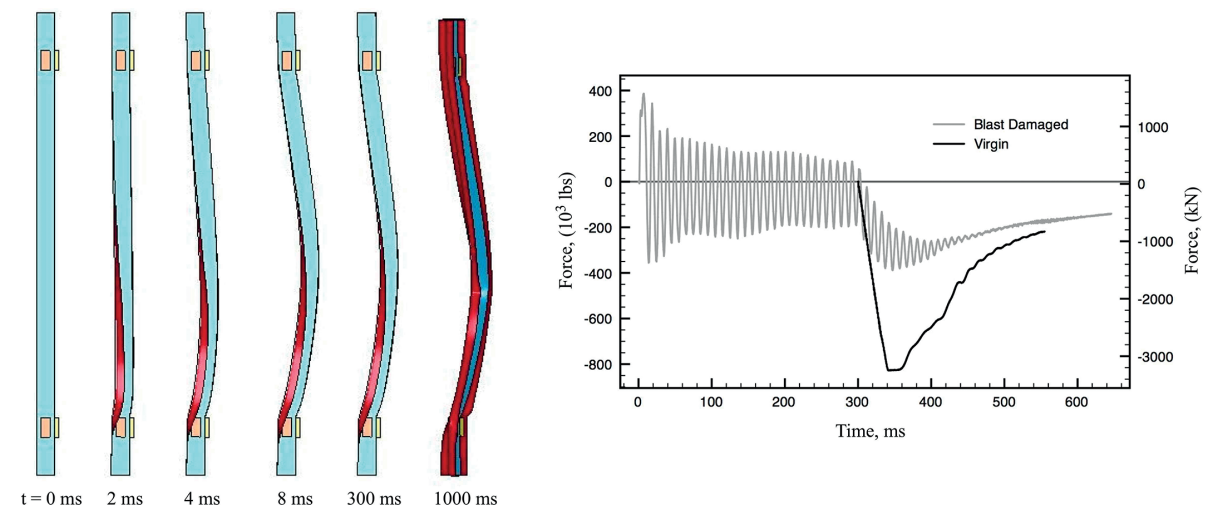

Figure 8: Strong axis model results.

axis model, the pressure load was applied to the web opposite the support. The loads were determined using BlastX and were distributed over the height of the column. Incident pressures were applied to the inside of the flanges to remain consistent with loads without clearing effects.

The 51 FEM cases were run according to the loading described above. Results from one representative case are included in this paper. This case was the nominal case. The standoff was $10 \mathrm{ft}$, the column had a depth of 14 inch $(35.6 \mathrm{~cm})$, a width-thickness ratio of 20, a thickness ratio of 1.6 and a $12 \mathrm{ft}(3.7 \mathrm{~m})$ length. A summary of the behavior through loading is given in Fig. 9(left). The first three frames show the column being loaded by the blast pressure load. The third frame shows the column being initially loaded axially and the last frame given the column buckling in a first mode response in the region with the most blast damage. The corresponding force diagram from this sample is given in Fig. 9(right). From this plot, the capacity of the column at failure was $2,164,000 \mathrm{lbs}(9,623 \mathrm{kN})$. This is compared to a virgin capacity test of $2,723,000 \mathrm{lbs}(12,113 \mathrm{kN})$. The associated CCR is 0.792 .

\section{MODEL TRAINING AND VALIDATION}

The structure of the neural network was set up to accommodate the five input parameters and the one output parameter and is given in Fig. 10. Initially, six hidden neurons were chosen for the first neuron layer. A logarithmic transfer function was used for both the hidden layer and the output layer because values of CCR are restricted from 0 to 1 . The Levenberg-Marquardt Algorithm (LMA) [14] was used as the training algorithm for this model and is built into the MATLAB Neural Network Toolkit [15]. The LMA provides a numerical solution for the minimization of a function created over a space of parameters by making the assumption that the underlying function being modeled by the neural network is linear. Based on this calculation, the minimum can be determined exactly in a single step. The calculated minimum is tested, and if the new error there is less, the algorithm moves the weights to this point [16].

The 51 sample input sets were randomly divided into 42 training data samples and 9 validation data samples. The LMA algorithm was trained with the training data sample as long as the network continued to reduce the error in the validation sample output. A plot of the mean square error versus iteration is given in Fig. 11. From this plot, it is evident that the model was learning and reducing error with each iteration.

The network can also be tested by using the initial training data (FEM inputs) as inputs and having the model determine the outputs. If the outputs are compared to the targets (FEM 

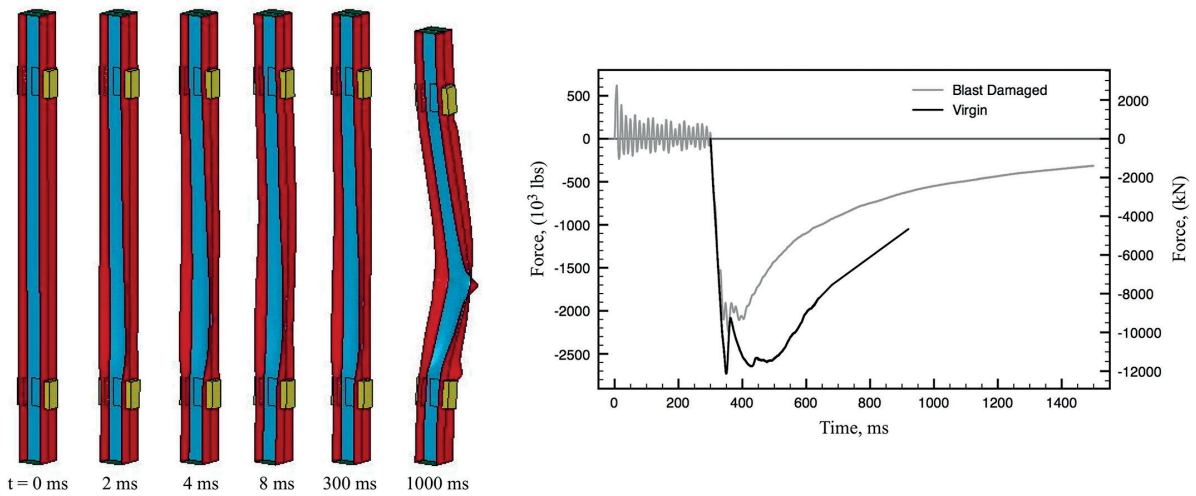

Figure 9: Weak axis model results.

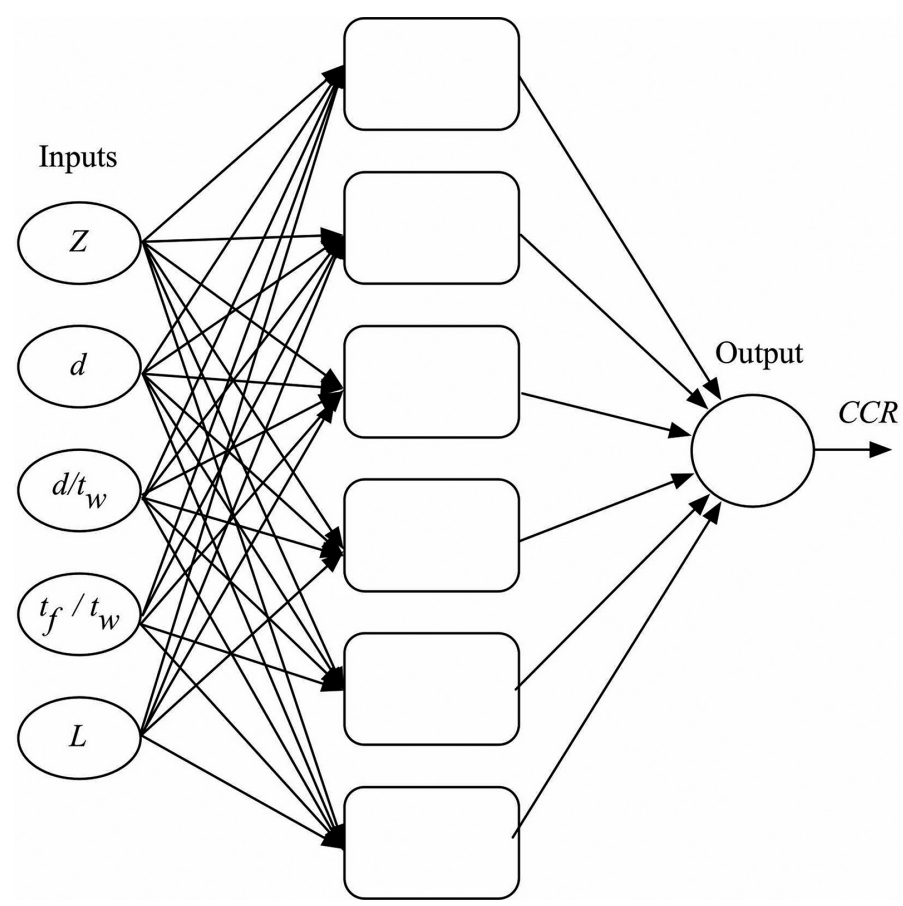

Figure 10: Steel column capacity neural network structure.

outputs), a regression analysis can be conducted. The results from this regression analysis are shown in Fig. 12. The $R$ value for the regression was 0.965 .

\section{MODEL TESTING}

The model was tested against one randomly selected column in each of the four depth ranges (W8, W10, W12, and W14). Each column was randomly assigned a standoff from 3 to $25 \mathrm{ft}$ and a column length from 8 to $25 \mathrm{ft}$. For each column, the FEM was run given the loading condition described by the standoff. The residual capacity of the column was calculated and compared to the output from the FRM. 


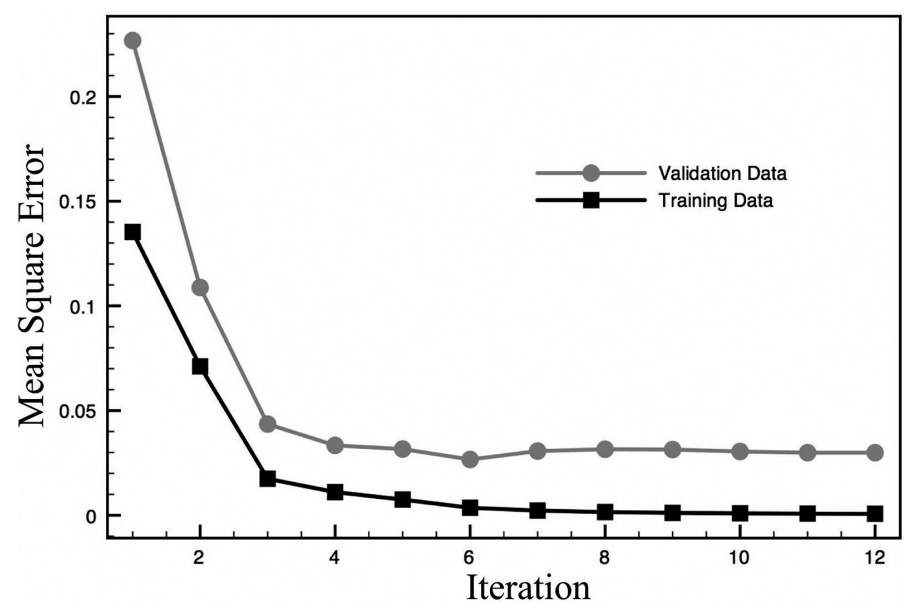

Figure 11: Neural network training performance.

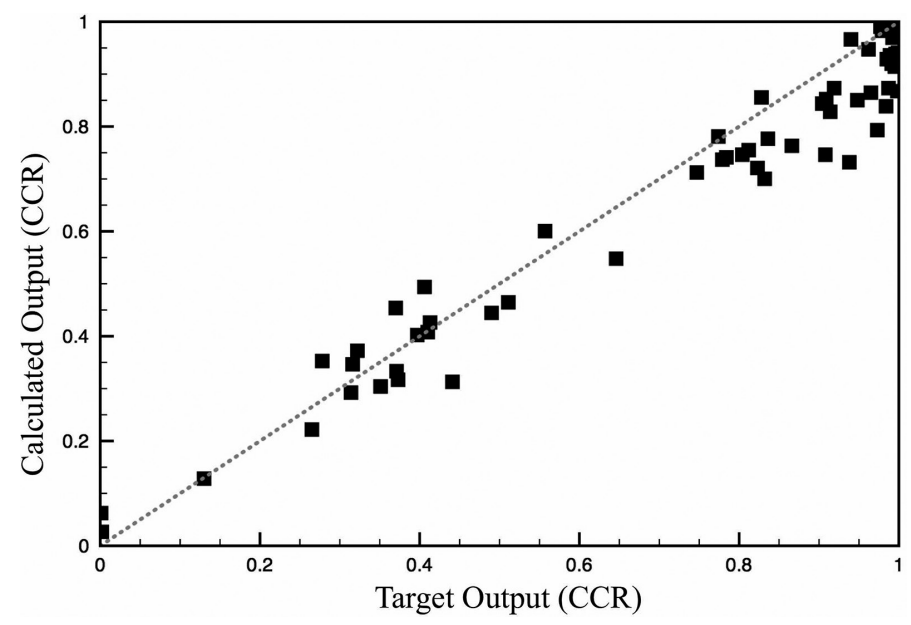

Figure 12: Comparison of target outputs and calculated outputs.

The results from the finite element runs for the eight test cases are summarized in Table 3. The column in Test 3 failed during the blast and therefore minimal residual capacity was computed. The results are compared to the CCR values calculated from the FRM along with the calculated error between the FRM and the FEM calculations for each of the randomly selected cases. The results from the FRM are all within $8 \%$ of the finite element calculations, which is well within an acceptable range for this application. Table 3 also summarizes the results from the FEM and from the FRM in the weak axis direction. All CCR values for the weak axis FRM are within 0.09 of the actual CCR. Adding additional data points is an option if a lesser error is required. 
Table 3: Results from randomly selected test cases.

\begin{tabular}{|c|c|c|c|c|c|c|c|c|}
\hline Test & W Section & $d$ (in) & $d / t_{\mathrm{w}}$ & $t_{\mathrm{f}} / t_{\mathrm{w}}$ & $L(\mathrm{ft})$ & FEM CCR & FRM CCR & Difference \\
\hline \multicolumn{9}{|c|}{ Strong Axis } \\
\hline 1 & W8x48 & 8.5 & 21.25 & 1.71 & 12 & 0.800 & 0.739 & $6.1 \%$ \\
\hline 2 & W10x112 & 11.36 & 15.05 & 1.66 & 21 & 0.924 & 0.906 & $1.8 \%$ \\
\hline 3 & W12x79 & 12.38 & 26.3 & 1.56 & 14 & 0.001 & 0.050 & $4.9 \%$ \\
\hline 4 & W14x99 & 14.16 & 22.73 & 1.6 & 10 & 0.853 & 0.884 & $3.1 \%$ \\
\hline \multicolumn{9}{|c|}{ Weak Axis } \\
\hline 5 & W8x40 & 8.25 & 22.92 & 1.5 & 18 & 0.300 & 0.387 & $8.7 \%$ \\
\hline 6 & W10x33 & 9.73 & 33.55 & 1.68 & 20 & 0.002 & 0.006 & $0.4 \%$ \\
\hline 7 & $\mathrm{~W} 12 \times 152$ & 13.71 & 15.76 & 1.61 & 22 & 0.231 & 0.231 & $0.0 \%$ \\
\hline 8 & W14x68 & 14.04 & 33.83 & 1.73 & 20 & 0.425 & 0.477 & $5.2 \%$ \\
\hline
\end{tabular}

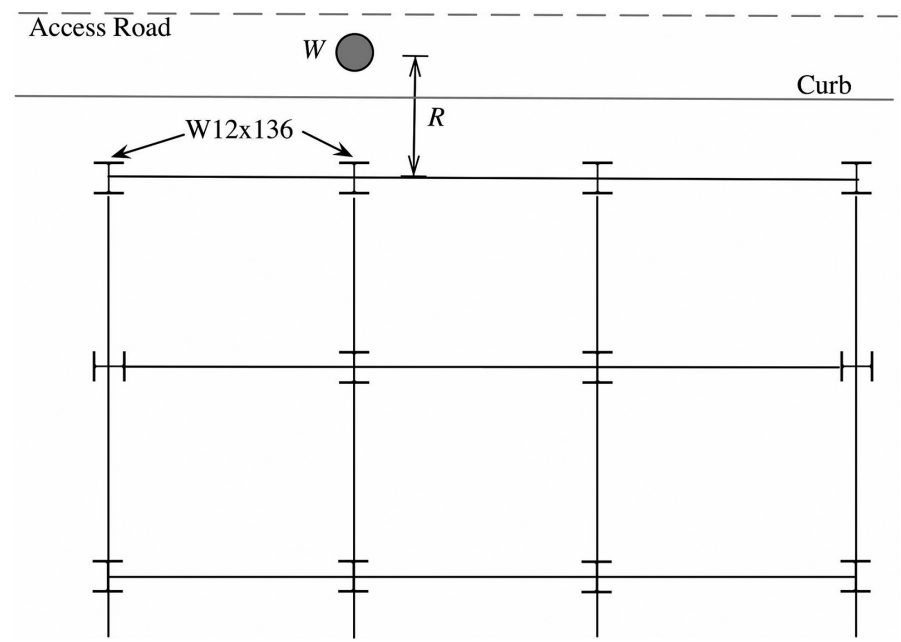

Figure 13: Fictitious building example.

\section{EXAMPLE}

The plan view for a fictitious building is shown in Fig. 13. The building is set next to an access road with vehicle traffic. A perimeter is needed to ensure that the building remains structurally sound, but minimizes the encroachment into the road. The columns are $14 \mathrm{ft}$ $(4.26 \mathrm{~m})$ tall and are W12' 136 sections. What is the minimum standoff needed to ensure that the structure can withstand at least $90 \%$ of its capacity given a vehicle-borne threat of $W \mathrm{lbs}$ of TNT (actual charge values intentionally omitted)?

Using the ANN created in this investigation, one can iterate on the standoff parameter and yield the results in Table 4. From the results, a standoff of $9 \mathrm{ft}(2.74 \mathrm{~m})$ is needed to ensure the building can withstand $90 \%$ of its capacity. One can get these results in a matter of seconds, without using blast software or creating an FEM. 
Table 4: Results from fictitious building example.

\begin{tabular}{lllllll}
\hline Charge & Standoff & $d$ & $d / t_{\mathrm{w}}$ & $t_{\mathrm{f}} / t_{\mathrm{w}}$ & $L$ & CCR \\
\hline$W$ & $7 \mathrm{ft}(2.13 \mathrm{~m})$ & 13.4 in $(39.0 \mathrm{~cm})$ & 16.97 & 1.58 & $14 \mathrm{ft}(4.26 \mathrm{~m})$ & 0.799 \\
$W$ & $8 \mathrm{ft}(2.44 \mathrm{~m})$ & 13.4 in $(39.0 \mathrm{~cm})$ & 16.97 & 1.58 & $14 \mathrm{ft}(4.26 \mathrm{~m})$ & 0.895 \\
$W$ & $\mathbf{9 f t}(\mathbf{2 . 7 4} \mathbf{~ m})$ & 13.4 in $(39.0 \mathrm{~cm})$ & 16.97 & 1.58 & $14 \mathrm{ft}(4.26 \mathrm{~m})$ & $\mathbf{0 . 9 5 6}$ \\
\hline
\end{tabular}

\section{CONCLUSIONS}

This paper responded to the growing necessity of a fast running analysis tool to predict column capacity that, unlike typical methods such as SDOFs, could take into account column effects that can vary with a standoff and charge size such as localized web buckling, flange bending, and web fracture. The model was created using a simplified FEM to populate a data set. The input parameters were chosen based on typical column dimensions as well as typical threat scenarios. The output, $C C R$, was selected to be a ratio of the damaged column capacity to the virgin column capacity. An ANN was used to predict the output parameter, and the methodology for the development including parameterization and model training was described.

The FRM was tested with four cases in both the strong and weak axis directions, which all produced responses close to that of the finite element runs. It was concluded that the model, using 51 finite element runs, is able to predict the response of relevant columns with an adequate level of confidence ( $<10 \%$ error). The methodology described can be further applied to various other column scenarios (i.e. pre-load, connections, material type) as well as applied to other loading scenarios where FRMs may be needed.

\section{REFERENCES}

[1] Biggs, J., Introduction to Structural Dynamics, McGraw-Hill: New York, 1864.

[2] Morrill, K., Malvar, L., Crawfor, J. \& Ferritto, J., Blast resistant design and retrofit of reinforced concrete columns and walls, Structures 2004, Nashville, TN, 22-26 May 2004, pp. 1-8, available at: http://ascelibrary.org/doi/abs/10.1061/40700(2004)154.

[3] Wu, K., Bing, L. \& Tsai, K., Residual axial compression capacity of localized blastdamaged RC columns. International Journal of Impact Engineering, 38(1), pp. 29-40, 2011. doi: http://dx.doi.org/10.1016/j.ijimpeng.2010.09.002

[4] Stewart, L.K., Experimental and computational methods for steel columns subjected to blast loads. WIT Transactions on the Built Environment, 126, pp. 157-168, 2012. doi: http://dx.doi.org/10.2495/SU120141

[5] Anderson, J., An Introduction to Neural Networks, The MIT Press: Cambridge, MA, 1995.

[6] Fausett, L., Fundamentals of Neural Networks, Prentice Hall: New York, NY, 1994.

[7] Mays, G.C. \& Smith, P.D., Blast Effects on Buildings - Design of Buildings to Optimize Resistance to Blast Loading, Thomas Telford: New York, 1995.

[8] American Institute of Steel Construction, Steel Construction Manual, 13th edn., American Institute of Steel Construction, 2006.

[9] Stewart, L.K., Computational modeling of steel columns subjected to experimentally simulated blasts, International Journal of Computational Methods and Experimental Measurements, 2(3), pp. 225-242, 2014. doi: http://dx.doi.org/10.2495/CMEMV2-N3-235-242 
[10] Livermore Software Technology Corporation, LS-DYNA Keyword User's Manual, LSTC: Livermore, CA, 2007.

[11] US Army Corps of Engineers, BlastX, 2002.

[12] Rigby, S., Tyas, A., Benneyy, T., Warren, J. \& Fay, S., Clearing effects on plates subjected to blast loads, ICE - Engineering and Computational Mechanics, 166(3), pp. 140-148, 2013.

[13] Smith, P.D., Rose, T. \& Saotongland, E., Clearing of blast waves from building facades, Proceedings of the Institution of Civil Engineers - Structures and Buildings, pp. 193-199, 1999. doi: http://dx.doi.org/10.1680/istbu.1999.31385

[14] Levenberg, K., A method for the solution of certain non-linear problems in least squares, The Quarterly of Applied Mathematics, 2, pp. 164-168, 1944.

[15] Demuth, H., Beale, M. \& Hagan, M., Matlab Neural Network Toolbox User's Guide, The MathWorks: Natick, MA, 1992.

[16] Press, W., Teukolsky, S., Vetterling, W. \& Flannery, B., Numerical Recipes in C: The Art of Computing, 2nd edn., Cambridge University Press: Cambridge, MA, 1992. 